\title{
Cytotoxic effect of Aeruginosin-865, capsaicin, and resveratrol on mouse cell lines of different origin
}

\author{
Ivana Veselá1, Jose Cheel$^{2}$, Jaroslava Tomenendálová1, Pavla Hamouzová ${ }^{1}$, \\ Pavel Hrouzek ${ }^{2}$, Śárka Stehlíková ${ }^{1}$, Kristína Řehákováa ${ }^{3}$ Jaroslav Doubek ${ }^{1}$ \\ ${ }^{1}$ University of Veterinary Sciences Brno, Faculty of Veterinary Medicine, Department of Physiology, \\ Brno, Czech Republic \\ ${ }^{2}$ Czech Academy of Sciences (CAS) v.v.i., Institute of Microbiology, Centre Algatech, \\ Třeboň, Czech Republic \\ ${ }^{3}$ University of Veterinary Sciences Brno, Faculty of Veterinary Medicine, Clinic Laboratory for Small Animals, \\ Brno, Czech Republic
}

Received September 4, 2020

Accepted August 31, 2021

\begin{abstract}
The aim of the study was to compare the effects of three natural bioactive compounds (and their combinations) on normal vs. tumour-transformed mouse cells. The cytotoxic effect of Aeruginosin-865 (Aer), capsaicin (Cap), resveratrol (Res) and their combinations was evaluated on normal hepatocytes (AML) and tumour cells derived from livers (Hepa) and kidneys (Renca). Various concentrations from 25 to $200 \mu \mathrm{M}$ of tested substances were used. Only the Aer + Res combination and a low concentration of Res had a significant cytotoxic effect on Hepa and Renca and no significant cytotoxic effect on AML. Cap had a significant cytotoxic effect on all tested cell lines, but tumour-derived cells showed higher resistance than AML. A significantly increased cytotoxicity was found in the combination of Cap + Res compared to each substance alone. All types of cells showed similar sensitivity to the cytotoxic effect of Cap + Res. Because of a possible hepatotoxic effect, we recommend further investigations into side-effects of Cap + Res. No cytotoxic effect was described in Cap + Aer or in Aer alone. Only substances with a significant cytotoxic effect on tumour cells and no cytotoxic effect on normal cells can be potentially used in anticancer treatment. According to the results, only Res or the combination of Aer + Res can be recommended for further evaluation in the process of new anticancer drug development. The potential hepatotoxic effect of Cap + Res can significantly limit the utilisation of these substances in anticancer treatment.
\end{abstract}

Alkaloids, anticancer treatment, natural bioactive compounds, phenolics, viability

Interesting biological properties such as antiproliferative, antioxidant or cytotoxic activities are offered by various natural substances. However, their potential utilization as therapeutic agents definitely requires determination of their safety profiles. The aim of this study was to describe a possible cytotoxic effect of Aeruginosin-865, capsaicin, and resveratrol on tumour cell lines in contrast to normal hepatocytes.

Aeruginosin-865 (Aer) was discovered in a strain of soil cyanobacterium (Nostoc sp.) (Kapuścik et al. 2013). This tetrapeptide has been shown to have anti-inflammatory effects mediated by inhibition of the NF- $\kappa B$ signalling pathway, which subsequently lead to inhibition of transcription of genes playing an important role in cell survival or inflammation progression (Kapuścik et al. 2013). Previous studies of Aer cytotoxicity in tumour or normal cells reported various results depending on the tested cell line. Whereas cytotoxicity was confirmed in tumour-transformed mouse fibroblasts (Veselá et al. 2018), no cytotoxic effect was observed in normal mouse fibroblasts (Veselá et al. 2018), in human hepatoma cells (Faltermann et al. 2016) or in human lung microvascular endothelial cells (Kapuścik et al. 2013).

Address for correspondence:

MVDr. Ivana Veselá, Ph.D.

University of Veterinary Sciences Brno

Palackého tř. 1946/1

61242 Brno

Czech Republic 
Capsaicin (Cap), trans-8-methyl-N-vanillyl-6-nonenamide, is an alkaloid found in the fruit of the Capsicum (Sharma et al. 2013). The effect of Cap on animals and humans has been widely studied, yielding promising results mainly in pain relief, inflammation, obesity and even cancer treatment or cancer prevention (Sharma et al. 2013). Most of the studies evaluating anticancer properties of Cap confirmed a selective inhibitory effect on cancer cells, whereas the viability and growth was not disrupted in normal cells (Bley et al. 2012; Sharma et al. 2013). Cell-cycle arrest, inhibition of cell growth and proliferation or apoptosis induction were proposed as the possible mechanisms underlying the anticancer effect (Clark and Lee 2016; Chapa-Oliver and Mejía-Teniente 2016). An anti-inflammatory effect of Cap has also been described (Reyes-Escogido et al. 2011). Cap had no cytotoxic effect on dermal fibroblasts (Lewinska et al. 2015), whereas fibrosarcoma cells were sensitive to Cap treatment (Ghosh and Basu 2010). Other studies reported very low sensitivity to Cap treatment in normal cells in contrast to tumour cells (Galati and O'Brien 2003; Bu et al. 2015; Veselá et al. 2018).

Resveratrol (Res) is a polyphenol synthesized by various plants such as grapevine, cranberries, broccoli or garlic. The disruption of mitochondrial transmembrane potential, increase in the production of oxygen radicals or increase in the intracellular calcium concentration have been suggested as mechanisms of anticancer effect but further investigations are necessary (Kim et al. 2009; Varoni et al. 2016; Ferraz da Costa et al. 2017). Res was shown to inhibit growth and proliferation in many cancer cell lines with limited cytotoxicity toward normal cells (Varoni et al. 2016; Ferraz da Costa et al. 2017; Veselá et al. 2018). Decreased cell viability and induced apoptosis were confirmed in fibrosarcoma cells (Lee and Kim 2011; Gweon and Kim 2013; Harati et al. 2015). The effect of Res on the cell viability was dose-dependent (Lee and Kim 2011; Gweon and Kim 2013; Veselá et al. 2018).

Based on the results of the previous studies, we hypothesized that cells of different origins would show a variable response to the effects of Aer, Cap, Res and their combinations. This is the first study in which the cytotoxic effects of Aer, Cap and Res were investigated on these mouse cell lines: normal hepatocytes (AML line), tumour-transformed hepatocytes (Hepa line) and tumour-transformed kidney cells (Renca line). The chosen cells are a suitable animal model of carcinogenesis. The effect of the above mentioned substances on chosen cell lines has not been studied before. Due to the increasing incidence of cancer in humans and animals, the obtained results contribute to the use of these natural bioactive substances as potential therapeutics in human and veterinary medicine.

Cell cultures

\section{Materials and Methods}

Normal hepatocytes (AML12 ATCC ${ }^{\circledR}$ CRL-2254TM) and tumour cell lines derived from liver (Hepa 1-6 ATCC $^{\circledR}$ CRL-1830 ${ }^{\mathrm{TM}}$ ) and kidney (Renca ATCC ${ }^{\circledR}$ CRL-2947 TM) tissues were tested. These adherent cell lines were cultured in the medium (normal hepatocytes: MEM (minimum essential medium) Alpha Medium from Thermo Fisher Scientific, Waltham, MA, USA; tumour cells: RPMI-1640 medium from Sigma-Aldrich, St. Louis, Missouri, USA) supplemented with $10 \%$ FBS (foetal bovine serum) (Sigma-Aldrich) and $1 \%$ penicillinstreptomycin (Sigma-Aldrich, St. Louis, Missouri, USA). Cell lines were placed in $5 \% \mathrm{CO}_{2}$ incubator at $37^{\circ} \mathrm{C}$. All analyses were performed in multiplicates (3-5) and were repeated 2-3 times.

Natural substances

Aeruginosin-865 (Aer) was obtained according to a previously reported procedure (Kapuścik et al. 2013) at the Laboratory of Algal Biotechnology, Institute of Microbiology, Czech Academy of Sciences in Třeboň, Czech Republic. Resveratrol (554325) (Res) and capsaicin (M2028) (Cap) were purchased from Sigma-Aldrich. DMSO (dimethyl sulphoxide) (D4540, Sigma-Aldrich) was used to dissolve the natural substances at maximal concentration of $20 \%$ DMSO to prepare stock solutions that were used for the preparation of exposure solutions. The individual exposure solutions were prepared by mixing the culture medium and the stock solution with final concentration of $0.1-1 \%$ DMSO. To exclude a potential cytotoxic effect of DMSO, a solvent control reaction with $0.1-2 \%$ DMSO was performed on untreated cells $(0.1-1 \%$ DMSO had no cytotoxic effect on all cell lines used (data not shown). 
Treatment schedule for cytotoxicity and lactate dehydrogenase test

To evaluate the cytotoxicity, lactate dehydrogenase (LDH) cytotoxicity assay kit (Thermo Fisher Scientific) was used according to the manufacturer's instructions. The cells were seeded on a 96-well plate at a density of $20 \times 10^{3}$ cells/well. After 24-h incubation, the cells were treated with Aer, Res, and Cap, and with their combinations (Cap + Res, Cap + Aer and Res + Aer), and were incubated with them for $24 \mathrm{~h}\left(37^{\circ} \mathrm{C}\right.$ in a $5 \% \mathrm{CO}_{2}$ atmosphere). Different concentrations of Aer, Cap, and Res were tested $(25 \mu \mathrm{M}, 50 \mu \mathrm{M}, 100 \mu \mathrm{M}$, and $200 \mu \mathrm{M})$. Cap + Res, Cap + Aer, and Res + Aer were used at the concentrations of $25+25 \mu \mathrm{M}, 50+50 \mu \mathrm{M}$, and $100+100 \mu \mathrm{M}$, respectively. The next day, the reaction mixture was added to each sample. The reaction was performed for $30 \mathrm{~min}\left(37^{\circ} \mathrm{C}\right.$; protected from light) and then stopped by the addition of the Stop Solution. The absorbance was read at $490 \mathrm{~nm}$ and $680 \mathrm{~nm}$ on a SynergyHT (BioTek, USA) instrument. LDH released from the cytosol of damaged cells induced tetrazolium conversion to a red formazan of intensity proportional to the amount of LDH released. A negative control (spontaneous LDH activity) was performed by treatment with sterile distilled water. A positive control (maximum LDH activity) was performed by treatment with lysis buffer $(\times 10)$ provided by the kit.

\section{Treatment schedule for viability and trypan blue test}

Viability of cells and their ability to establish new colonies (proliferation test) after treatment was tested at $100 \mu \mathrm{M}$ concentrations of Cap, Res, and Aer, or $0.1 \%$ DMSO (control). Control cells were incubated only in appropriate medium. After 24-h incubation (24-well plate, $12 \times 10^{4}$ cells/well) the cells were evaluated, passaged (determination of viability was performed using trypan blue exclusion test) and compared with the seeded number. Then, the cells were seeded again to appropriate complete medium (at the same concentration of $12 \times 10^{4}$ cells/well or all cells if the obtained number of cells was lower). After $24 \mathrm{~h}$ the number of cells and their viability were compared with the seeded concentration.

\section{Statistical analysis}

The significance of the difference between each concentration vs the control treated sample was evaluated using the MedCalc statistical software. $T$-test was applied for statistical analysis. The level of significance was $P<0.05$.

\section{Results}

No significant cytotoxicity of Aer compared to the control group was observed in the tested cell lines at tested concentrations. Cap showed a significant cytotoxic effect in all the tested cell lines (both tumour cells and normal hepatocytes) at the concentration of $200 \mu \mathrm{M}$. In normal hepatocytes it showed cytotoxicity as early as at the concentration of $50 \mu \mathrm{M}$, whereas in cancerous cell lines at $200 \mu \mathrm{M}$. Thus, the cytotoxic effect of Cap was demonstrated at low concentrations in AML, whereas only in up to $\times 4$ higher concentrations in Hepa and Renca. Therefore, the cytotoxicity of capsaicin was significantly higher in normal hepatocytes than in tumour-derived cells (Hepa and Renca). Res showed significant cytotoxicity in tumour-derived cell lines at all the tested concentrations (i.e. as low as $25 \mu \mathrm{M}$ ), whereas in the normal AML line, cytotoxicity occurred only at the highest concentrations (100 and $200 \mu \mathrm{M})$.

The combination of Cap + Res showed higher cytotoxicity than Res alone in all the tested cells (in AML and Hepa from $50 \mu \mathrm{M}$, in Renca even from $25 \mu \mathrm{M}$ ). Thus, the combination of Cap + Res had a significant cytotoxic effect on both normal hepatocytes and tumour cells (Hepa and Renca), in all cases at low concentrations. The cytotoxic effect of the Aer + Res combination was also evident in the Hepa and Renca tumour cell lines. The difference between the normal AML cells and the control treated sample was non-significant. The Cap + Aer combination showed lower cytotoxicity than the substances alone, in all lines and at all tested concentrations. More detailed results are described in Figs 1, 2, and 3.

Cap inhibited proliferation in both tumour cell lines (Hepa and Renca), whereas Aer only in the kidney-derived tumour cells (Renca). The decrease in the proliferative activity was particularly evident if cells were incubated in $100 \mu \mathrm{M}$ Res. More details are described in Fig. 4. The subsequent ability to establish new colonies after passage was also reduced. 


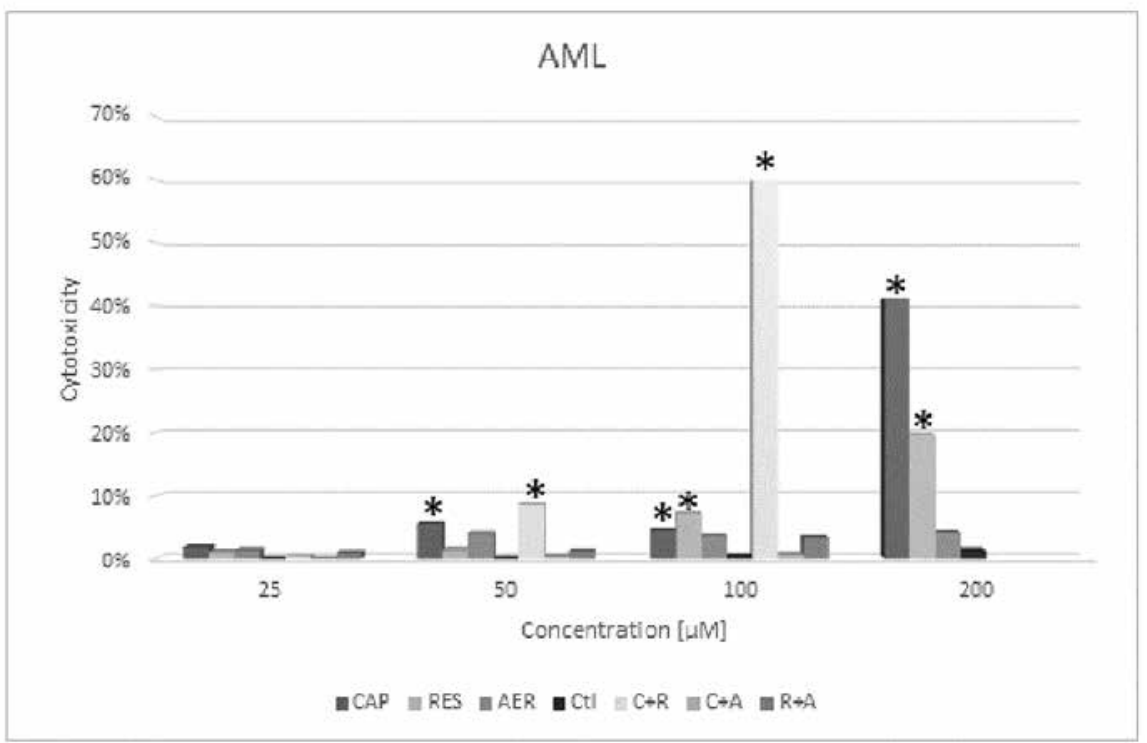

Fig. 1. Cytotoxicity of capsaicin, resveratrol, Aeruginosin- 865 and their combinations on mouse AML cell line derived from normal hepatocytes. Controls represent untreated cells incubated with $1 \%$ dimethyl sulphoxide. The experiment was repeated $\times 5$ and the data in the graph represent the mean of the repetition. * indicates $P<0.05$ in comparison to the control group. CAP - capsaicin, RES - resveratrol, AER - Aeruginosin-865, Ct1 - control, $\mathrm{C}+\mathrm{R}$ - capsaicin + resveratrol, $\mathrm{C}+\mathrm{A}$ - capsaicin + Aeruginosin-865, R+A - resveratrol + Aeruginosin-865

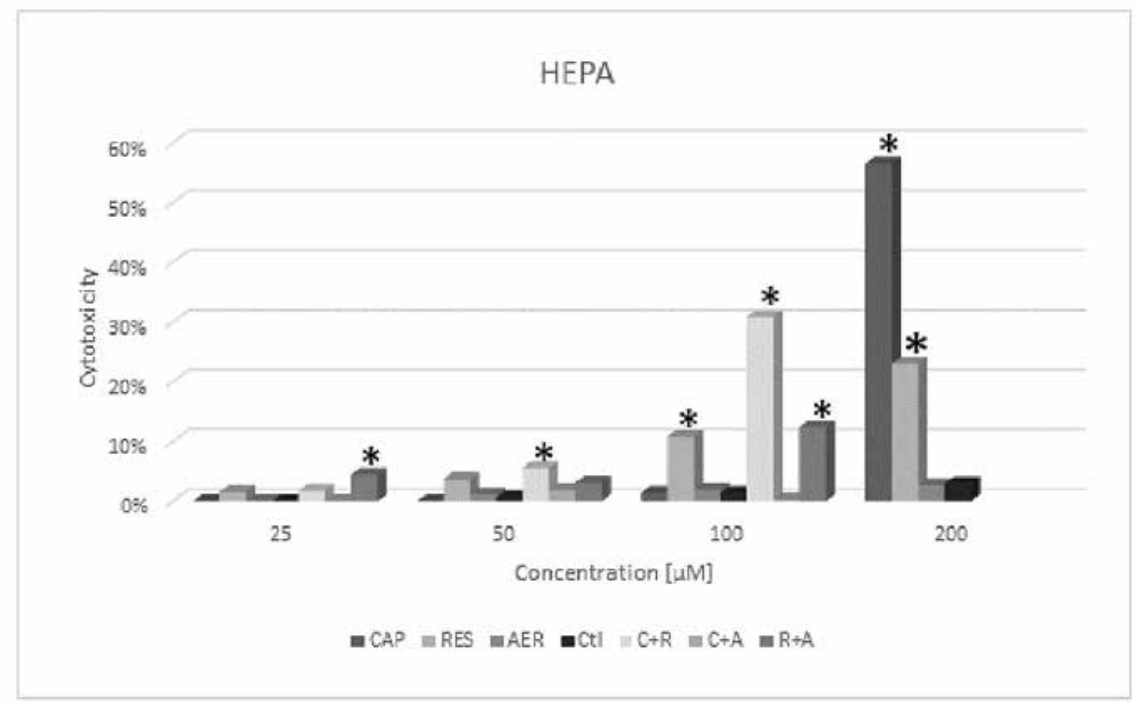

Fig. 2. The cytotoxicity of capsaicin, resveratrol, Aeruginosin- 865 and their combinations on mouse Hepa cell line derived from tumour-transformed hepatocytes. Controls represent untreated cells incubated with $1 \%$ dimethyl sulphoxide. The experiment was repeated $\times 5$ and the data in the graph represent the mean of the repetition. * indicates $P<0.05$ in comparison to the control group. CAP - capsaicin, RES - resveratrol, AER - Aeruginosin-865, Ctl - control, C+R - capsaicin + resveratrol, C+A - capsaicin + Aeruginosin-865, $\mathrm{R}+\mathrm{A}$ - resveratrol + Aeruginosin- 865 


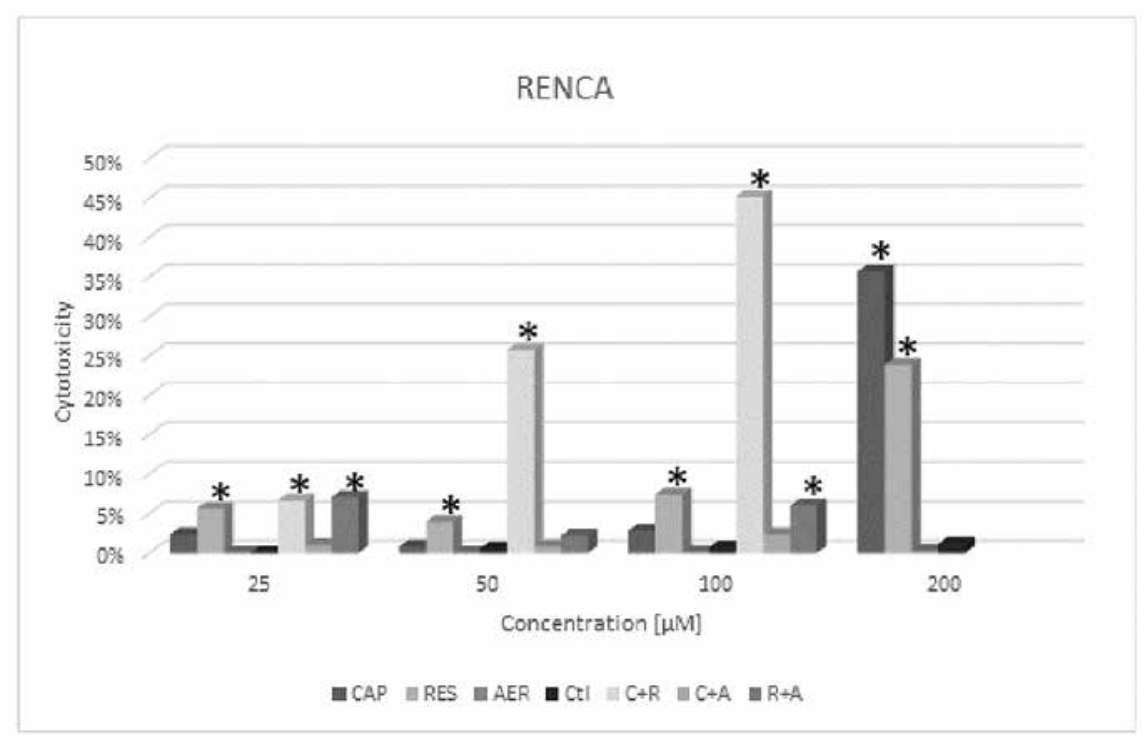

Fig. 3. The cytotoxicity of capsaicin, resveratrol, Aeruginosin- 865 and their combinations on mouse Renca cell line derived from tumour-transformed kidney cells. Controls represent untreated cells incubated with $1 \%$ dimethyl sulphoxide. The experiment was repeated $\times 5$ and the data in the graph represent the mean of the repetition. * indicates $P<0.05$ in comparison to the control group. CAP - capsaicin, RES - resveratrol, AER - Aeruginosin-865, Ctl - control, C+R - capsaicin + resveratrol, C+A - capsaicin + Aeruginosin-865, $\mathrm{R}+\mathrm{A}$ - resveratrol + Aeruginosin- 865

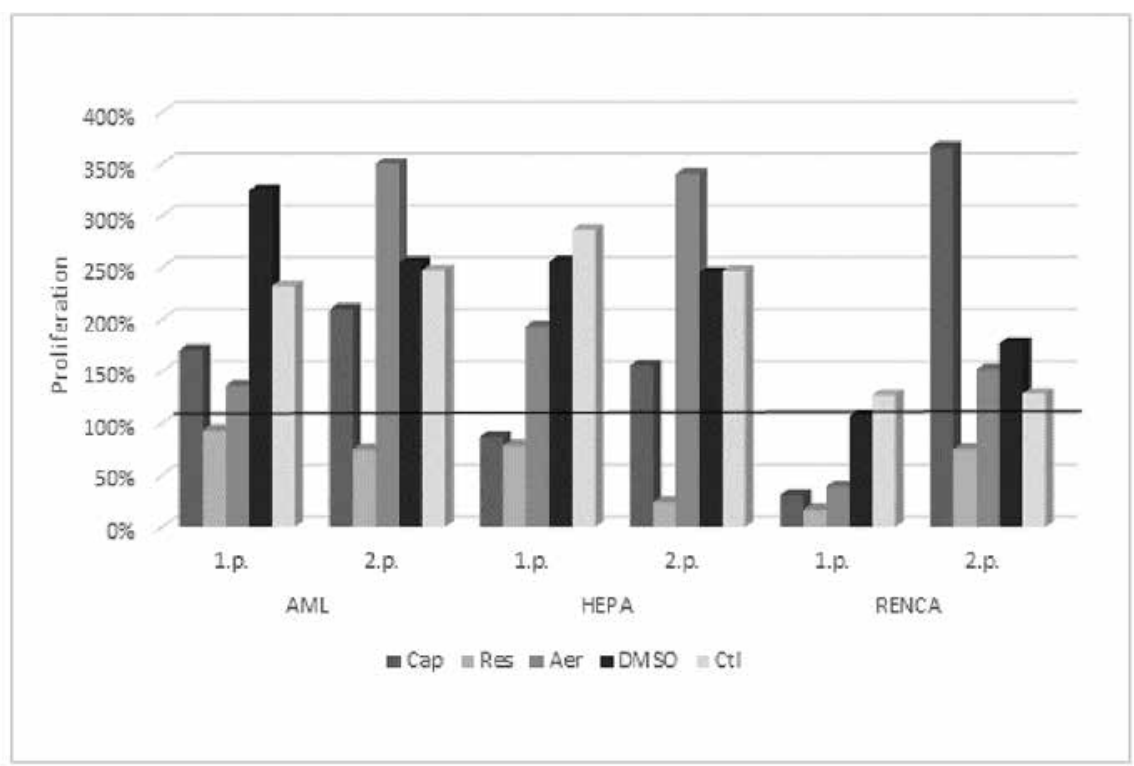

Fig. 4. Proliferation of mouse normal hepatocytes, liver-derived tumour cells, and kidney-derived tumour cells after treatment with capsaicin, resveratrol, and aeruginosin in contrast to controls. 1.p. and 2.p. - first and second passage, AML - normal hepatocytes, HEPA - liver-derived tumour cells, RENCA - kidney-derived tumour cells, Cap - capsaicin, Res - resveratrol, Aer - Aeruginosin-865, DMSO - dimethyl sulphoxide, Ctl - control 


\section{Discussion}

No cytotoxic effect of Aer was found in tumour cells (Hepa and Renca), similarly as was previously reported in human hepatoma cells (Faltermann et al. 2016). On the contrary, tumour-transformed mouse fibroblasts were sensitive to the cytotoxic effect of Aer (Veselá et al. 2018). This difference was probably caused by the variety of tested tumour cells.

As to the normal cells, no cytotoxic effect of Aer on hepatocytes (AML line) was observed. Previous studies reported resistance to the Aer treatment in human lung microvascular endothelial cells (Kapuścik et al. 2013) or in normal fibroblasts (Kapuścik et al. 2013; Faltermann et al. 2016; Veselá et a. 2018). On the other hand, the cytotoxicity of Aer was previously described in normal liver- and kidney-derived cells of fallow deer, but only at the highest used concentration of $200 \mu \mathrm{M}$ (Veselá et al. 2018). Thus, the cytotoxic effect of Aer could not be clearly excluded. Interspecies differences probably appear.

Cap showed a significant cytotoxicity as early as at the concentration of $50 \mu \mathrm{M}$ in AML, whereas in tumour cells at $200 \mu \mathrm{M}$. No effect on the viability was reported in dermal fibroblasts, whereas mouse embryonic fibroblasts were sensitive to the Cap treatment, with the first cytotoxic effect observed at a dose of $50 \mu \mathrm{M}$ (Lewinska et al. 2015) - so at the same level as we described in AML.

The widely reported apoptotic or growth inhibitory effects of Cap, which were selective for tumour cells and left normal cells unharmed (Ghosh and Basu 2010; Bley et al. 2012; Lewinska et al. 2015) differ greatly from our results. In our study, the cytotoxic effect of Cap was observed only at the concentration of $200 \mu \mathrm{M}$ in both tumour-derived cell lines (Hepa and Renca). Thus, the normal cell lines were more sensitive to the Cap treatment than tumour cells, in contrast to some previous studies performed on different tumour cells (Galati and O'Brien 2003; Ghosh and Basu 2010; Bley et al. 2012; Bu et al. 2015; Lewinska et al. 2015; Veselá et al. 2018).

The effect of Cap on Hepa was the same as on Renca. A previously reported higher resistance to the Cap treatment in kidney-derived cells from fallow deer compared to liverderived cells (Veselá et al. 2018) indicates that the effect of Cap varies among different cells and different species. This can be the reason why our results vary from some other studies (Galati and O'Brien 2003; Ghosh and Basu 2010; Bley et al. 2012; Bu et al. 2015; Lewinska et al. 2015; Veselá et al. 2018). Nevertheless, it is difficult to compare these results with our study as we did not use two types of normal cells, we only compared tumour cells derived from the two organs. Further investigations should be performed to elucidate these properties.

Res showed significant cytotoxicity in both tumour cell lines at all tested concentrations (i.e. as low as $25 \mu \mathrm{M}$ ), whereas in normal hepatocytes (AML line), cytotoxicity occurred only at the highest concentrations $(100$ and $200 \mu \mathrm{M})$. Thus, according to our results, the cytotoxic activity of Res could potentially be used for the development of new anticancer drugs. Res was shown to inhibit growth and proliferation in many cancer cell lines with the limited cytotoxicity toward normal cells (Varoni et al. 2016; Ferraz da Costa et al. 2017; Veselá et al. 2018). Res decreased cell viability and induced apoptosis also in fibrosarcoma cells (Lee and Kim 2011; Gweon and Kim 2013; Harati et al. 2015; Veselá et al. 2018). Res did not induce cytotoxic effects in normal mouse fibroblasts (Veselá et al. 2018), however, we found them in normal hepatocytes (albeit only at the highest concentrations). Similarly, as we described in AML, a significant cytotoxic effect of Res was found in liver- and kidney-derived cells from fallow deer (Veselá et al. 2018). Based on our results, Res could potentially be used to develop anticancer drugs. Therefore, possible side-effects (hepatotoxicity) should be studied more deeply.

We confirmed a synergic anti-tumour effect of Cap and Res previously reported in human tumour pancreatic cells (Vendrely et al. 2017). The combination of Cap + Res 
caused apoptosis in human melanoma cells (Kim 2012) and even radiosensitized some of pancreatic tumour cells (Vendrely et al. 2019). On the other hand, a neuroprotective effect of this combination was described on mouse cell lines (Lee et al. 2012). Nevertheless, this combination showed significant cytotoxicity not only in tumour cells but also in normal hepatocytes. Because of the possible hepatotoxic effect, we recommend to perform further investigations into the side-effects of this combination.

This study is the first description of the cytotoxic effect of the Aer + Res combination. Significant cytotoxicity was found in both tumour cells but not in the AML line. Thus, it could potentially be used to develop anticancer drugs. Low- and high-dose cytotoxicity was found in the Aer + Res combination.

The Cap + Aer combination showed lower cytotoxicity than the substances alone in all lines and at all tested concentrations. No data for comparison are available from previous studies.

Comparison of the cytotoxic effects of the tested substances on kidney-derived and liver-derived tumour cells brought interesting results. The effects of Cap, Aer, Cap + Aer, and Res + Aer were the same in both cell lines. On the other hand, the effect of Res and $\mathrm{Cap}+\mathrm{Res}$ varied greatly. The cytotoxicity of Res started at the concentration of $25 \mu \mathrm{M}$ in Renca, whereas at $100 \mu \mathrm{M}$ in Hepa. The combination of Cap + Res had a cytotoxic effect from $25 \mu \mathrm{M}$ in Renca, but from $50 \mu \mathrm{M}$ in Hepa. To conclude, the cytotoxic effect of these natural substances varies among cell lines and is significantly affected by the dose.

A decrease in proliferative activity was found in all cells incubated with Res at the concentration of $100 \mu \mathrm{M}$. This corresponds to the cytotoxic effect of Res (described above).

Only substances with a significant cytotoxic effect on tumour cells and no cytotoxic effect on normal cells could potentially be used for the development of new anticancer drugs. Therefore, this utilisation can be expected only in Res and also in the combination of Aer + Res. A possible hepatotoxic effect of the Cap + Res combination needs further investigations.

\section{Conflict of Interest}

The authors declare that there is no conflict of interest.

\section{Acknowledgments}

The authors thank IAPG AS CR, v.v.i. for a loan of the SynergyHT reader. This work was supported by the Internal Grant Agency of the University of Veterinary Sciences Brno, Grant No. 106/2018/FVL.

\section{References}

Bley K, Boorman G, Mohammad B, McKenzie D, Babbar S 2012: A comprehensive review of the carcinogenic and anticarcinogenic potential of capsaicin. Toxicol Pathol 40: 847-873

Bu HQ, Cai K, Shen F, Bao XD, Xu Y, Yu F, Pan HQ, Chen CH, Du ZJ, Cui JH 2015: Induction of apoptosis by capsaicin in hepatocellular cancer cell line SMMC-7721 is mediated through ROS generation and activation of JNK and p38 MAPK pathways. Neoplasma 62: 582-591

Chapa-Oliver AM, Mejía-Teniente L 2016: Capsaicin: from plants to a cancer-suppressing agent. Molecules 21: 931

Clark R, Lee SH 2016: Anticancer properties of capsaicin against human cancer. Anticancer Res 36: 837-843

Faltermann S, Hutter S, Christen V, Hettich T, Fent K 2016: Anti-inflammatory activity of cyanobacterial serine protease inhibitors Aeruginosin 828A and Cyanopeptolin 1020 in human hepatoma cell line Huh7 and effects in zebrafish (Danio rerio). Toxins 8: 219-233

Ferraz da Costa DC, Fialho E, Silva JL 2017: Cancer chemoprevention by resveratrol: the p53 tumor suppressor protein as a promising molecular target. Molecules 22: 1014

Galati G, O’Brien PJ 2003: Cytoprotective and anticancer properties of coenzyme Q versus capsaicin. Biofactors 18: $195-205$

Ghosh AK, Basu S 2010: Fas-associated factor 1 is a negative regulator in capsaicin induced cancer cell apoptosis. Cancer Lett 287: 142-149

Gweon EJ, Kim SJ 2013: Resveratrol induces MMP-9 and cell migration via the p38 kinase and PI-3K pathways in HT1080 human fibrosarcoma cells. Oncol Rep 29: 826-834 
Harati K, Slodnik P, Chromik AM, Goertz O, Hirsch T, Kapalschinski N, Klein-Hitpass L, Kolbenschlag J, Uhl W, Lehnhardt M, Daigeler A 2015: Resveratrol induces apoptosis and alters gene expression in human fibrosarcoma cells. Anticancer Res 35: 767-774

Kapuścik A, Hrouzek P, Kuzma M, Bártová S, Novák P, Jokela J, Pflüger M, Eger A, Hundsberger H, Kopecký J 2013: Novel Aeruginosin-865 from Nostoc sp. as a potent anti-inflammatory agent. Chem Bio Chem 14: 2329-2337

Kim MY 2012: Nitric oxide triggers apoptosis in A375 human melanoma cells treated with capsaicin and resveratrol. Mol Med Rep 5: 585-591

Kim MY, Trudel LJ, Wogan GN 2009: Apoptosis induced by capsaicin and resveratrol in colon carcinoma cells requires nitric oxide production and caspase activation. Anticancer Res 29: 3733-3740

Lee SJ, Kim MM 2011: Resveratrol with antioxidant activity inhibits matrix metalloproteinase via modulation of SIRT1 in human fibrosarcoma cells. Life Sci 88: 465-472

Lee JG, Yon JM, Lin C, Jung Y, Jung KY, Nam SY 2012: Combined treatment with capsaicin and resveratrol enhances neuroprotection against glutamate-induced toxicity in mouse cerebral cortical neurons. Food Chem Toxicol 50: 3877-3885

Lewinska A, Chochrek P, Smolag K, Rawska E, Wnuk M 2015: Oxidant-based anticancer activity of a novel synthetic analogue of capsaicin, capsaicin epoxide. Redox Rep 20: 116-125

Reyes-Escogido M de L, Gonzalez-Mondragon EG, Vazquez-Tzompantzi E 2011: Chemical and pharmacological aspects of capsaicin. Molecules 16: 1253-1270

Sharma SK, Vij AS, Sharma M 2013: Mechanisms and clinical uses of capsaicin. Eur J Pharmacol 720: 55-62

Varoni EM, Lo Faro AF, Sharifi-Rad J, Iriti M 2016: Anticancer molecular mechanisms of Resveratrol. Front Nutr 3: 8

Vendrely V, Amintas S, Noel C, Moranvillier I, Lamrissi I, Rousseau B, Coulibaly S, bedel A, MoreauGaudry F, Buscail E, Chiche L, Belleannée G, Dupin C, Dabernat S 2019: Combination treatment of resveratrol and capsaicin radiosensitizes pancreatic tumor cells by unbalancing DNA repair response to radiotherapy towards cell death. Cancer Lett 451: 1-10

Vendrely V, Peuchant E, Buscail E, Moranvillier I, Rousseau B, Bedel A, Brillac A, de Verneuil H, Moreau-Gaudry F, Dabernat S 2017: Resveratrol and capsaicin used together as food complements reduce tumor growth and rescue full efficiency of low dose gemcitabine in a pancreatic cancer model. Cancer Lett 390: 91-102

Veselá I, Celá-Kolísková P, Kuchařová V, Tomenendálová J, Kováčová V, Pikula J, Repková B, Rapekta P, Hrouzek P, Cheel J, Doubek J 2018: Cytotoxic effect of aeruginosin-865, resveratrol and capsaicin on mouse fibroblasts and cells derived from fallow deer. Nat Prod Commun 13: 205-208 